\title{
From Thermal Energy to Kinetic Energy: Droplet Motion Triggered by the Leidenfrost Effect
}

\author{
Guanqi Wang a, Jonathan R. McDonough a , Vladimir Zivkovic a, Teng Long ${ }^{\mathrm{b}}$, Steven Wang c,* \\ ${ }^{a}$ School of Engineering, Newcastle University, Newcastle Upon Tyne, UK \\ ${ }^{\mathrm{b}}$ School of Engineering, University of Cambridge, UK \\ 'Department of Mechanical Engineering, City University of Hong Kong, Hong Kong \\ *Corresponding author: steven.wang@ cityu.edu.hk
}

\begin{abstract}
When a liquid is dropped on a surface significantly hotter than the liquid's boiling point, a vapour film will form beneath the droplet creating an insulation layer sufficient enough to prevent the droplet from rapidly boiling. This phenomenon is known as the Leidenfrost effect, and enables droplets to survive for up to several minutes before fully evaporating. Solids are similarly able to levitate due to sublimation. Furthermore, a liquid droplet placed on a heated flat surface moves randomly, but on a ratcheted substrate, will self-propel and move unidirectionally along the ratchets. Such a system with no other external energy fields applied is designated a Leidenfrost self-propulsion device, first introduced by Linke et al. Given the ability of such an arrangement to effectively convert thermal energy into kinetic energy, numerous studies have subsequently attempted to understand and refine the control of motion of the levitated droplets/solids. This review addresses the fundamental understanding of this 'heat-to-motion' mechanism, where the main focus is conversion of thermal energy into kinetic energy through the unique Leidenfrost self-propulsion mechanism. Potential applications of Leidenfrost selfpropulsion devices are also discussed, including a brief outlook for the future of this research field.
\end{abstract}

Key words: Leidenfrost effect, self-propulsion, heat-to-motion, energy conversion 


\section{Introduction}

Several no-moving-parts (i.e. 'passive') liquid transport phenomena rely in some way on the cohesive and adhesive properties of the liquid; no active control is required. For example, the photosensitive system shown in figure 1a demonstrates liquid motion on a modified surface via a surface energy gradient in response to the application of graded lighting intensity ${ }^{[1]}$. This is an implementation of the Marangoni effect ${ }^{[2]}$, which describes the motion of a liquid resulting from local gradients of surface tension (cohesive forces) by either thermal treatment or chemical modification to the surface ${ }^{[3,4]}$. Adhesion, on the other hand, has the ability to induce capillary imbibition (the absorbance of one liquid into another). A recent study used the phenomenon to self-propel slugs inside a tube, where a 'bislug' composed of two different liquids created continuous asymmetry of the Laplace pressure that resulted in motion (figure $1 \mathrm{~b})^{[5]}$.

(a)

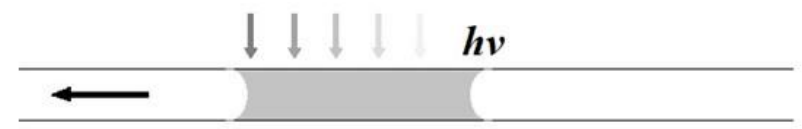

(b)

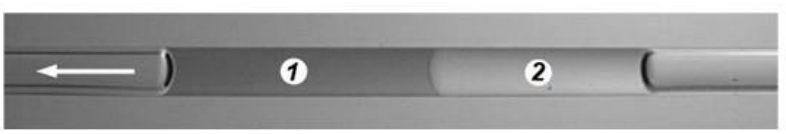

(c)

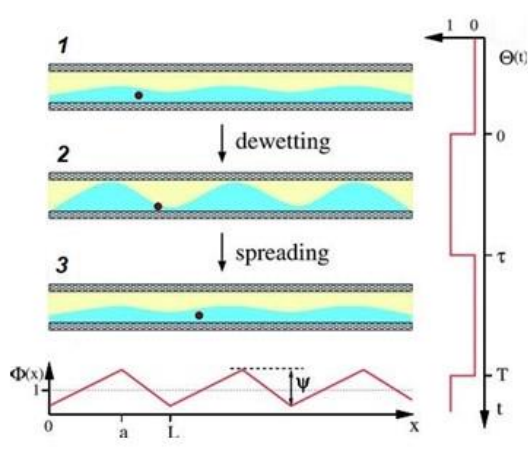

(d)

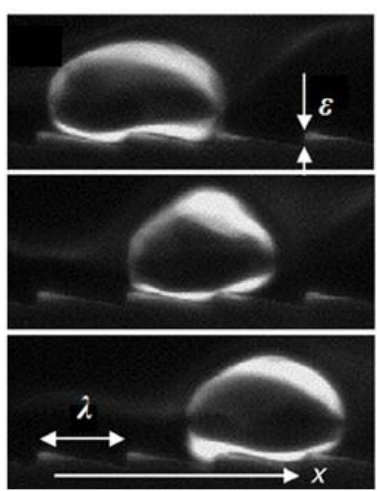

Fig. 1. 'Passive' methods to induce liquid motion | (a) Marangoni flow induced by gradient light intensity ${ }^{[1]},(b)$ imbibition induced flow ${ }^{[5]}$, (c) liquid spreading in an electric field on a ratcheted surface $^{[6]},(d)$ Leidenfrost self-propulsion on a heated ratcheted surface ${ }^{[7]}$

Water droplets can also be moved in a preferential direction by externally oscillating hydrophobic surfaces textured with specific micro/nano-features ${ }^{[8,9]}$. And, the rebound direction of water droplets deposited on superhydrophobic surfaces can be adjusted using micro/nanoscale patterns imbued onto the surface ${ }^{[10,11]}$. More recently, droplets have shown the capability to continuously bounce on smooth superhydrophobic surfaces with restitution coefficients greater than 1 (rebound height increases until a new equilibrium is reached), which is driven by the vaporisation of the liquid in low pressure environments ${ }^{[12]}$. In all of these examples, the rebound direction mainly depends on the local wetting characteristics rather than a simple dependence on the morphology ${ }^{[11]}$. Further, these droplet motion methods produce limited displacement distances and velocities, and the fabrication of the 
micro/nanoscale surface features is time-consuming and expensive, making these droplet control techniques impractical.

Alternatively, droplet transport can be induced on ratcheted surfaces using either an electric field (figure 1c) ${ }^{[6,13]}$, or via the use of the Leidenfrost effect (figure 1d). These ratcheted surfaces also exist in nature. For example, Shorebirds have beaks containing ratcheted capillaries that are able to transport water in combination with mechanical tweezering motion ${ }^{[14]}$. In these examples, it is the combination of an external energy source with the surface and liquid properties that introduces the anisotropy necessary for propulsion. Modern material science also introduces new methodologies to modify the wetting/dewetting properties of these ratcheted surfaces, opening up further opportunities to enhance and control droplet motion.

The common observation of seemingly random motion of water droplets on a hot kitchen surface/pan is an example of Leidenfrost induced motion. However, Linke et al. ${ }^{[7]}$ recently reported Leidenfrost induced controlled motion of liquid droplets. They found that a droplet of liquid would be propelled unidirectionally on a saw-tooth ratcheted surface, provided the substrate was heated above the droplet's Leidenfrost state (figure 1d). Here, a vapour layer insulates and levitates the droplet from the surface, enabling motion for a considerable life time with velocities on the order of $\sim 0.1 \mathrm{~m} / \mathrm{s}^{[15,16]}$. This has inspired several studies to understand the origins and fundamentals of this phenomenon, leading to further developments in micro/nano fabrication technologies as well as several interesting applications. The purpose of this mini review is to highlight the state-of-the-art in this research area to identify the present gaps in knowledge and corresponding future research directions.

\section{Varieties of Leidenfrost Self-Propulsion System \\ 2.1 Leidenfrost Phases}

Theoretically, any vaporisable material has the ability to self-propel on a ratcheted surface as long as the Leidenfrost state is obtained. The Leidenfrost effect occurs when a liquid or solid "contacts" a surface significantly hotter than the boiling or sublimating points respectively, resulting in the formation of a vapour layer that insulates and suspends the liquid/solid from the surface. Note, we use the word 'contact' to describe the interaction between the Leidenfrost body and the surface for simplicity, though the resulting vapour film actually prevents direct contact. This drastically increases the lifetime of said liquid/solid, because conduction across the vapour film becomes the major contributor to the heat transfer rate, though the lifetime also depends on the physical properties of the levitated body (i.e. boiling or sublimation point) and the surface roughness ${ }^{[17]}$.

The initial study by Linke et al. ${ }^{[7]}$ demonstrated Leidenfrost self-propulsion of R134a $(1,1,1,2$ tetrafluorethane), liquid nitrogen, acetone, methanol, ethanol, hexadecane and water droplets on a ratcheted surface ${ }^{[7]}$. The droplet sizes needed to be maintained around their capillary lengths, $l_{c}$, because liquid slugs with lengths of tens of millimetres were found to break apart into smaller droplets (unless they were enclosed between adjacent walls). Note, the capillary length is defined in equation 1 , where $\gamma, \rho_{L}$ and $g$ are the surface tension and liquid density at the boiling point, and gravity respectively ${ }^{[18]}$. The propulsion of solids (e.g. dry ice) on a similar ratcheted surface was then later observed by Guillaume et al. ${ }^{[19]}$, which inspired a debate on the exact origin of the mechanism for motion (to be introduced in Section 3). Beyond the study with pure matter, Ali et al. ${ }^{[20]}$ designed a levitated solid cart using water droplets as frictionless 'wheels' and a metal platelet as the load-bearing substrate, as shown in figure 2 . The propulsion force, indicated by either terminal velocity or inclination angle of the surface, depended on both water volume beneath the cart and the mass of the supported load.

$$
l_{c}=\left(\frac{\gamma}{\rho_{L} g}\right)^{1 / 2}
$$




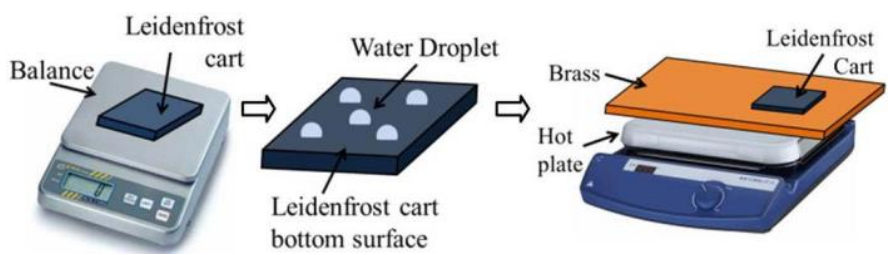

Fig. 2. Experimental set up of the Leidenfrost cart self-propulsion. Water droplets evenly distributed at the bottom of a plate act as "wheels" that drag the plate as they propel along the heated ratcheted surface $^{[20]}$

In the review, 'Leidenfrost dynamics', Quéré ${ }^{[15]}$ discussed the physical characteristics of a Leidenfrost liquid droplet on a flat surface, including the static description of the droplet shape, the stability of the liquid/vapour interface, the evaporation analysis of the vapour film and the film thickness calculation. However, an important area lacking any significant research is the study of the deformation characteristics of a droplet as it interacts with ratchets. This highlights the first area worthy of future study.

\subsection{Substrate Design}

Linear ratchets (that move the droplet along a single axis) initially received the most attention, which included the elucidation of the ratchet scale on droplet motion. Later, more novel substrate designs were considered that showed good flexibility towards the control of a droplet's motion. This section discusses the novel characteristics of these designs.

Linke et al.'s study ${ }^{[7]}$ showed that the ratchet geometry had little influence over the propulsion characteristics when linear ratchets were manufactured at millimetre scales. For substrates with a ratchet period $(\lambda)$ of $1-3 \mathrm{~mm}$ and a height $(\varepsilon)$ of $0.1-0.3 \mathrm{~mm}$ (see figure $1 \mathrm{~d}$ ), the terminal velocities of water droplets around their capillary sizes were typically $\sim 0.05 \mathrm{~m} \mathrm{~s}^{-1}$. Ok. et al. ${ }^{[21]}$ subsequently reduced the ratchet geometries to micron and sub-micron scales using micromilling and polymer optical grating respectively, and manufactured ratchets with periods $(\lambda)$ of $800 \mathrm{~nm}, 15 \mu \mathrm{m}, 75 \mu \mathrm{m}, 150 \mu \mathrm{m}$ and 1.5 $\mathrm{mm}$. They observed that a small water droplet ( $5 \mu \mathrm{L}$ volume) moved at much higher velocities $(0.1-0.4$ $\mathrm{m} \mathrm{s}^{-1}$ ) on these ratchets at temperatures much lower than typically needed for the Leidenfrost regime (figure 3). 


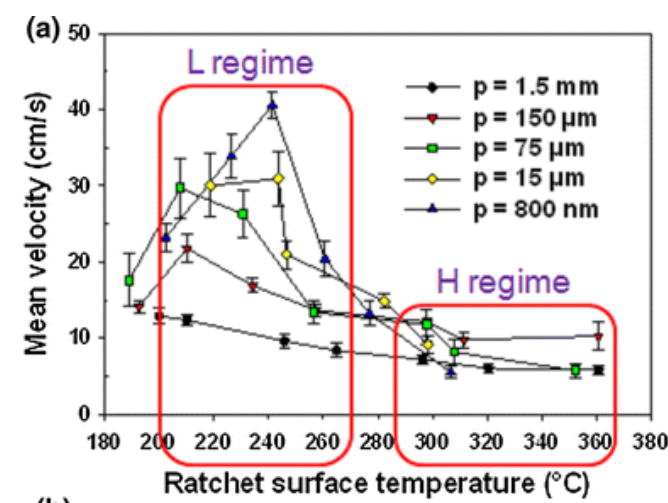

(b)

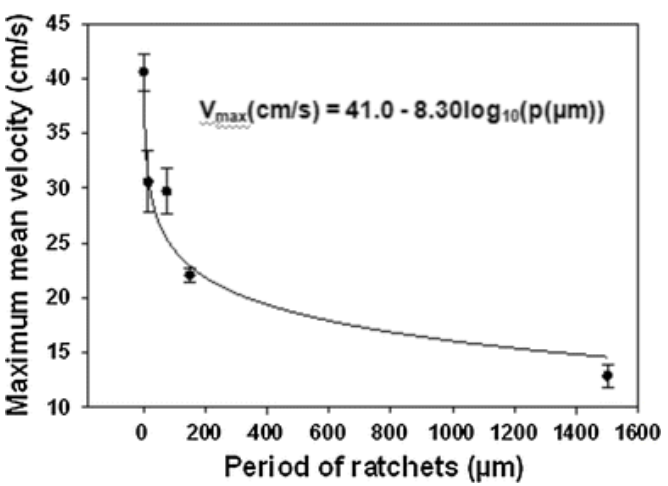

Fig. 3. Mean droplet velocity as a function of (a) surface temperature, and (b) ratchet period. The mean velocity was taken as the average distance travelled divided by the time. In the high temperature

$(H)$ regime, the droplet was in the Leidenfrost state where a fully developed vapour film existed, producing consistent mean velocities $\left(\sim 0.1 \mathrm{~m} \mathrm{~s}^{-1}\right)$. In the low temperature $(L)$ regime, the droplet partially contacted the substrate, and the mean velocities were higher than the H regime (reaching a maximum of $\left.\sim 0.4 \mathrm{~m} \mathrm{~s}^{-1}\right)^{[21]}$

Specifically, they identified a threshold temperature $\left(T_{T h}\right)$ to trigger droplet propulsion that was lower than the corresponding Leidenfrost point on the specific surface. This had also been observed by Linke et al. ${ }^{[7]}$, who noted that higher droplet acceleration with higher terminal velocities existed in a lower temperature regime, even though the insulating vapour layer was not fully developed. This low temperature regime (figure 3a) corresponds to the transition boiling regime ${ }^{[22]}$. Here, the liquid partially contacts the surface, which enhances the heat transfer rate, leading to an increased evaporation rate that enhances propulsion. The local surface contact was also evidenced by Alex et al. $\left[{ }^{23]}\right.$ by monitoring the sound produced by the droplets. Little sound was recorded in the Leidenfrost regime, whereas the transition boiling regime produced a notable amplitude in the sound signal, enabling both regimes to be differentiated. However, the enhanced velocities on the micro-ratchets were only observed for droplets smaller than their capillary lengths ${ }^{[21,24]}$. Ok et al. ${ }^{[21]}$ hypothesised that the partial conversion from the vertical impact velocity to the horizontal velocity must also be considered, which seems reasonable when considering the quasi-spherical shape of those small droplets. The small contact area enables them to rotate in response to any external energy field. In summary, these studies show that a 'positive' propulsion effect is produced on micro-scaled ratchets, because the increased surface roughness is also accompanied by a raised Leidenfrost point leading to the transition boiling regime ${ }^{[25-27]}$.

Agapov et al. ${ }^{\left[28,{ }^{29]}\right.}$ further reduced the ratchet scale by fabricating tilted nanopillars. They used glancing-angle anisotropic reactive ion etching (RIE) of a thermally dewet platinum (Pt) mask to create the pillars (see figure 4). Water droplets deposited on these nano-etched surfaces either moved randomly or against the tilted pillars. Equation 2 defines the Weber number, $W e$, where $V$ is the impact velocity and $D$ is the droplet diameter. The uncontrollable motion mainly occurred if the droplet was impacted on the nano-etched surfaces without an adequate Weber number. The Weber number defines the ratio of inertia to surface tension. Thus, small Weber numbers do not classify the droplet rebound into the conventional Leidenfrost self-propulsion regime (with directed vapour flow beneath the droplet). For 
water droplets with sufficient Weber number ( 100-1100, dependent on the temperature), the nanopillars represent a new approach to induce and control droplet motion based analogously on the Leidenfrost effect. The rebound direction can be controlled if the surface wettability becomes asymmetric (preferential wetting in one direction compared to another), in accordance with previous demonstrations on textured hydrophobic surfaces ${ }^{[8,10,11,30]}$. The directed rebound on the nanopillared surfaces is then a consequence of three phenomena. Firstly, the contact of liquid and solid upon impact ${ }^{[31,32]}$ that causes the liquid to enter the transition boiling regime ${ }^{[22,33,34]}$. Secondly, the directed liquid spreading on the surface because of its preferential wettability ${ }^{[9,35]}$. Finally, the rebound characteristics intrinsic to the small droplet ${ }^{[31]}$. As for the uncontrollable motion for a gently dispersed droplet (smaller Weber number), the nanopillars were not 'asymmetric enough' to define a vapour flow direction between the droplet and surface; Ok et al. ${ }^{[21]}$ postulate that the levitated droplet interacts with the nanoscaled substrate as if it were just a flat surface.

$$
W e=\frac{\rho_{L} V^{2} D}{\gamma}
$$

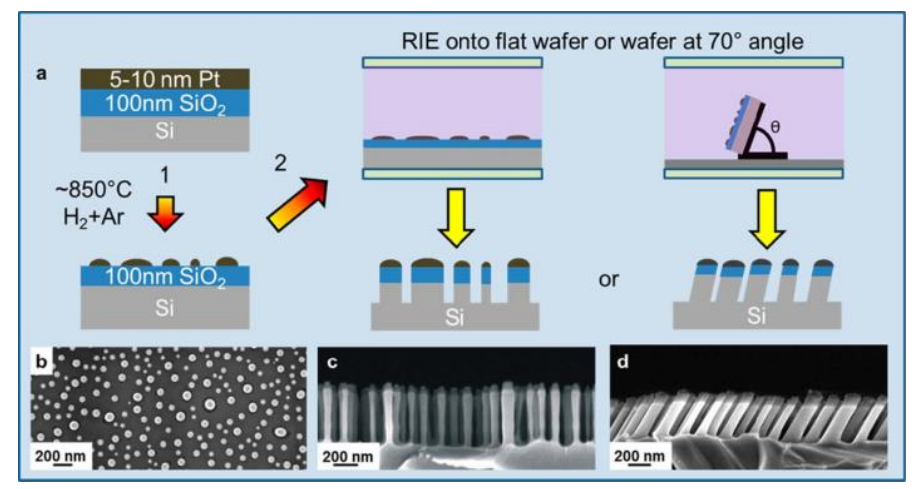

Fig. 4. (a) Fabrication sequence to create nonopillar arrays. A $5 \mathrm{~nm}$ layer of Pt was deposited onto a silicon ( $\mathrm{Si}$ ) wafer with $100 \mathrm{~nm}$ of thermally grown silicon oxide $\left(\mathrm{SO}_{2}\right)$. (al) Annealing in $\mathrm{H}_{2}$ :Ar at $\sim 850^{\circ} \mathrm{C}$ led to the formation of circular Pt islands due to metal dewetting. (a2) RIE etching of wafer positioned horizontally or a wafer tilted at $70^{\circ}$ relative to the carrier wafer resulted in straight or tilted arrays of nanopillars, respectively. | (b) SEM image of circular Pt islands formed as a result of dewetting a $5 \mathrm{~nm}$ thick Pt film. | (c-d) Cross-sectional SEM image of nanopillary array ${ }^{[28]}$

Hydrophobic surface coatings have shown the ability to drastically reduce the operating temperatures needed to induce the Leidenfrost self-propulsion effect ${ }^{[36,37]}$, because the hydrophobicity will significantly increase the contact angle (and therefore reduce adhesion) between a liquid-solid interface ${ }^{[37]}$. According to Guillaume et al. ${ }^{[36]}$, a surface temperature as low as $125^{\circ} \mathrm{C}$ could produce a droplet velocity of $\sim 0.064 \mathrm{~m} \mathrm{~s}^{-1}$, which is comparable to the velocities reported by Linke et al. at $250{ }^{\circ} \mathrm{C}{ }^{[7]}$. Surprisingly, the critical temperature to observe the self-propulsion effect could also be lower than the liquid's boiling point, albeit with a negligible associated velocity. This hydrophobic surface coating technique may therefore unlock the capability to recover lower grade/quality waste heat for energy harvesting applications.

In addition to the linear ratcheted surfaces, Thomas et al. ${ }^{[38]}$ constructed a concentrically ratcheted disc that caused water droplets to follow radial/elliptical trajectories as shown in figure 5. The ratchets were manufactured with a period of $\lambda=1.5 \mathrm{~mm}$ and height of $\varepsilon=0.3 \mathrm{~mm}$. They designated this as a Leidenfrost trap. The acceleration and velocity were found to oscillate between two corresponding peak values (around $\pm 4 \mathrm{~m} \mathrm{~s}^{-2}$ and $\pm 0.2 \mathrm{~m} \mathrm{~s}^{-1}$ respectively), both of which were significantly larger than previous results on linear ratchets at comparable millimetre scales. However, the droplets were less stable in this trap. Even droplets around the capillary length scale were observed to enter a spinning state if lateral momentum was also introduced at the point of deposit; this caused the droplets to become unstable and break apart. 
(a)

(b)
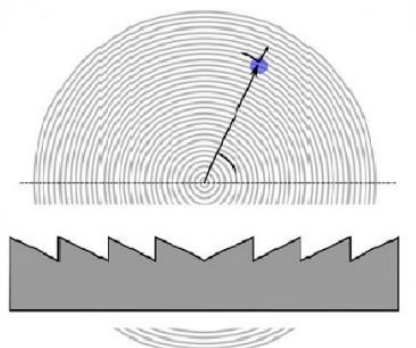

(c)

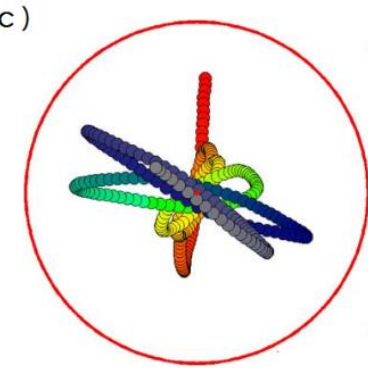

Fig. 5. (a-b) Design of a ratchet trap, (c) Trajectory of Leidenfrost water droplets on the trap. Imparting initial angular momentum caused the droplet to move with an elliptical trajectory (radial and tangential motion). Without any initial angular momentum, the droplet followed a pseudo $1 D$ orbit (radial motion only) ${ }^{[38]}$

In contrast to the limitations of the Leidenfrost trap, another propulsion example was demonstrated on a flat surface by tilting a solid Leidenfrost body with a small weight as shown in figure $6 \mathrm{a}^{[39]}$. The absence of the ratchets made this transportation mode more efficient in two ways. First, this approach is theoretically more scalable because the absence of the ratchets eliminates the potential for the levitated object to strike the steps. Second, the absence of the ratchets also reduces the apparent friction associated with the energy loss of the deformed liquid (figure $6 \mathrm{~b}$ ) ${ }^{[40]}$, meaning the acceleration phase for a Leidenfrost solid is longer, which enhances the terminal velocity (increasing the energy conversion efficiency).

(a)

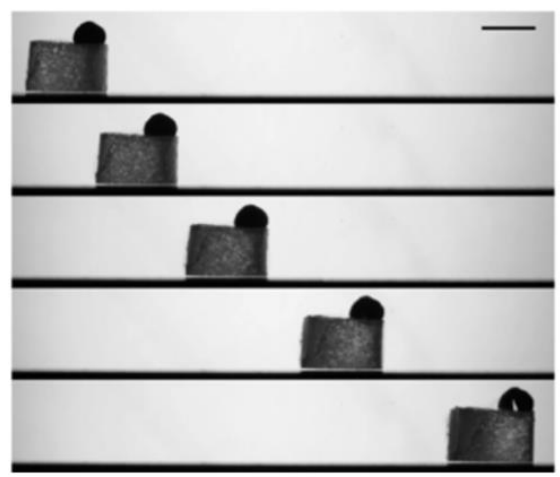

(b)

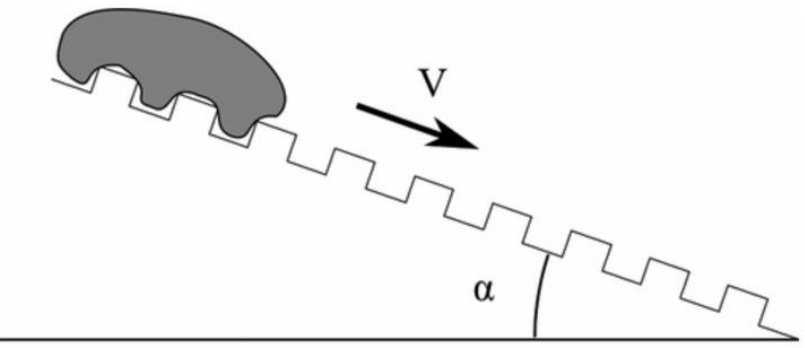

Fig. 6. (a) Leidenfrost self-propulsion on a flat surface ${ }^{[39]}$, (b) liquid deformation into the grooves ${ }^{[40]}$. A small weight was added on one side of a sample of dry ice creating an asymmetry that produced a vapour flow that then induced motion ${ }^{[39]}$

Related to the motion in figure 6a, liquid droplets can also glide/self-propel on the surface of a second liquid, as long as the viscosity ${ }^{[41]}$ and boiling point ${ }^{[42,43]}$ are significantly different. For instance, Gauthier et al. ${ }^{[44]}$ deposited various liquid drops (ethanol, silicone oil, propanol, butanol, pentanol, and water) onto the surface of a liquid nitrogen bath. Here it was the evaporation of the liquid substrate that generated the insulating vapour layer. They observed through experiment and modelling that the film thickness of this vapour layer differed between the front and trailing edges of the droplets. Therefore, like the dry ice in figure $6 \mathrm{a}$, it is this asymmetry that induces motion. Thus, this approach demonstrates another method for frictionless droplet transport, though the drops need to be around capillary length are able to levitate ${ }^{[42]}$ and the velocities are more limited (around $0.2 \mathrm{~m} \mathrm{~s}^{-1}$ ) ${ }^{[43,44]}$. Further, the vapour film thins over time as the drops cool down, increasing the friction causing them to decelerate. Though the drops can also remain suspended after they freeze, where they eventually sink once the temperature of the drop and bath equilibrate.

Recently, Bouillant et al. ${ }^{[45]}$ found yet another way in which small Leidenfrost droplets self-propel on a smooth rigid substrate. Small droplets $(R<1.5 \mathrm{~mm})$ were observed to self-rotate and create an asymmetric internal flow that resulted in a symmetry-breaking that cause the small droplets to tilt 
(similar to the artificial treatment in ${ }^{[39]}$ ). In contrast, large droplets failed to self-propel because the convective-driven internal flows were symmetric. The theoretical framework of this "legendary mobility" emphasises the importance of the vapour film thickness, and this may inspire other novel designs of self-propulsion devices according to the authors' expectations (e.g. a solid substrate with temperature gradient modification).

A somewhat related phenomenon has also been observed to convert thermal to kinetic energy, which may provide an alternative strategy for building a waste heat engine. Hydrogels are three-dimensional hydrophilic polymer networks that can absorb relatively large volumes of water making them remarkably elastic. Usually, when elastic materials impact solid surfaces, the kinetic energy is converted into elastic potential energy and a small amount of heat as the material deforms. It is the dissipation of this heat that results in energy loss, causing the kinetic energy to decline over time (figure 7a). However, hydrogel spheres impacted on hot surfaces have shown the ability to continually rebound for several minutes (figure $7 \mathrm{~b}$ ) ${ }^{[46]}$. In this case, the energy loss is compensated by an overpressure deformation caused by the evaporation of the absorbed liquid and resulting ejection of the vapour. Thus, the droplets will bounce provided there is sufficient liquid remaining. Restitution coefficients (ratio of relative speeds after and before collision) greater than unity have even been observed, meaning hydrogel spheres placed on heated surface will seemingly spontaneously bounce from a resting state ${ }^{[47]}$. The same effect can also be triggered at room temperature by reducing the pressure around the hydrogel ${ }^{[12]}$, since it is evaporation of the liquid that provides the energy for mechanical bouncing.

However, there is some debate between the mechanisms proposed in ${ }^{[46]}$ and ${ }^{[47]}$. Scott et al. ${ }^{[46]}$ studied the continuous bouncing that resulted from dropping the spheres onto an already heated surface, where they identified high-frequency oscillations $(2 \mathrm{kHz})$ in the overpressure/vapour release as the driving cause (similar to a steam engine). Whereas Pham et al. ${ }^{[47]}$ studied the 'spontaneous' jumping of the hydrogels from rest as the surface heats up, and identified a single build-up of overpressure as the mechanism. Alternatively, mechanical energy can be created via the collision of two hydrogel balls in a microwave field, because the contact region will create an intense electromagnetic hotspots that causes a similar internal overpressure via evaporation ${ }^{[48]}$.
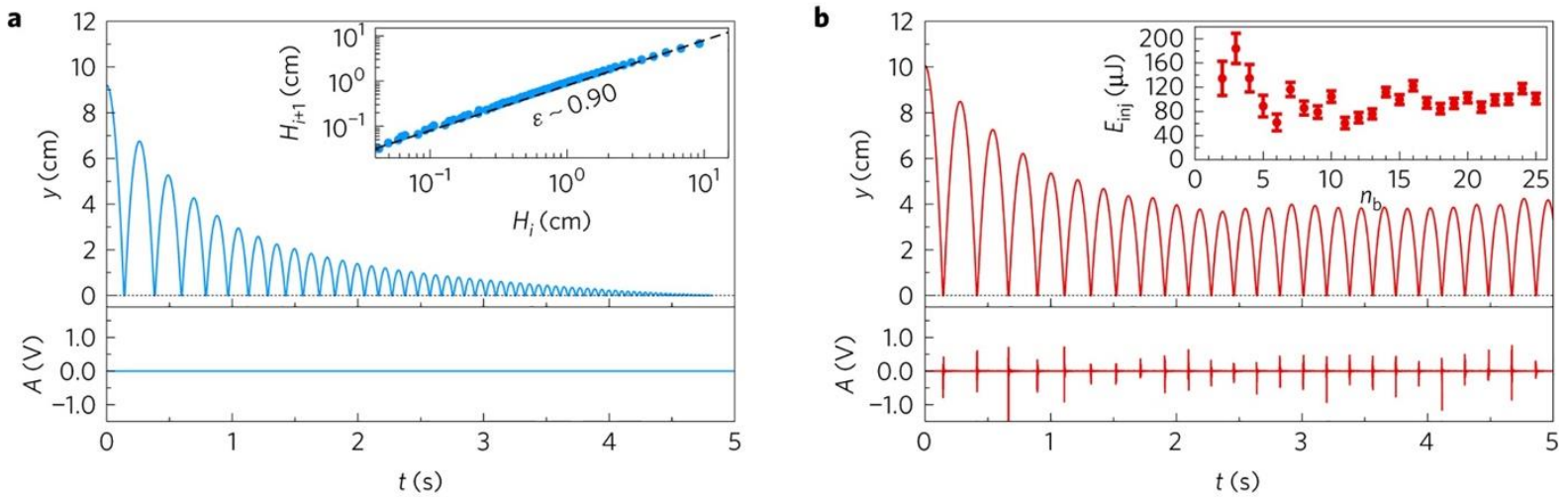

Fig. 7. Vertical displacement (top) and audio (bottom) vs time graphs for (a) hydrogel sphere bouncing on a cold surface, and (b) hydrogel sphere bouncing on a heated surface. A hydrogel deposited on a cold surface has a restitution coefficient less than 1 (so it loses energy over time). On a

heated surface, the restitution coefficient can be equal to, or even greater than, 1 because of the energy released during evaporation, allowing the hydrogel to continuously bounce for a considerable period of time (this provides the basis for a basic heat engine) ${ }^{[46]}$

\section{Dynamics and Friction}

The exact mechanism for the Leidenfrost self-propulsion and the friction exerted by the ratchets has been the subject of debate for some time. Some of the proposed theories have been experimentally validated, whilst others have been supported by numerical analysis. This section of the review will outline and compare these proposed mechanisms, including in-depth discussion of the self-propulsion phenomenon itself. 


\subsection{Viscous Stress Induced by Laplace Pressure}

Initially, Linke et al. ${ }^{[7]}$ proposed that the droplet is driven by a viscous force exerted by the rectified vapour flow between the droplet and the ratcheted surface. They compared the pressure difference in the vapour film using the Laplace pressure function (equation 3), where $\Delta p$ is the pressure difference between the droplet's internal pressure and the external pressure in the vapour film and $r$ is the local radius of the curvature of the droplet. Using this model, they observed a pressure gradient along the vapour layer by considering the negative and positive curvature differences between points $A$ and $B_{2}$ (shown in figure 8). This pressure differential between point $A$ and point $B_{2}$ subsequently induces a vapour flow down the slow slope of the steps, while part of the vapour escapes laterally into and out of the plane of figure 8 (see figure 10 for clarification). Therefore, a net 'forward' driving force causes the vapour to flow 'forward' in each ratchet period. Because of the interfacial viscous force (i.e. no-slip boundary condition), this vapour flow then 'drags' the droplet in the same direction.

$$
\Delta p=\frac{\gamma}{r}
$$
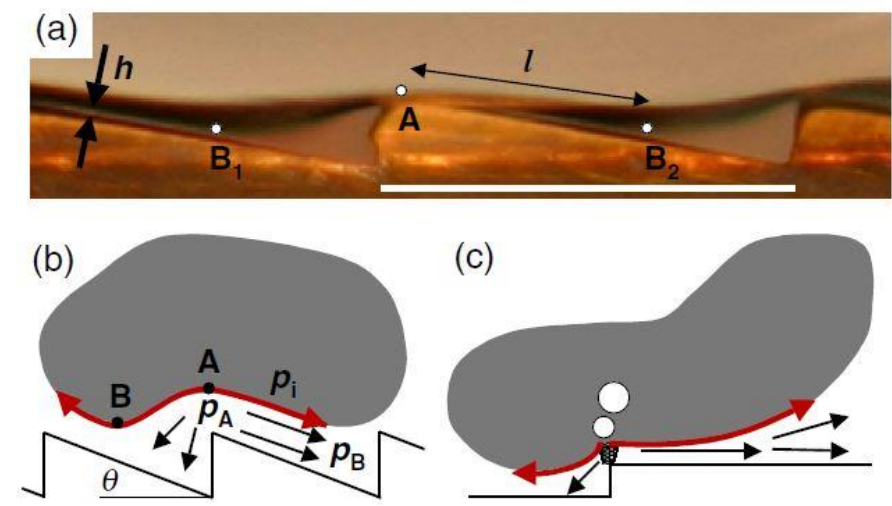

Fig. 8. Pressure difference in the vapour film caused by the droplet's deformation. The different local curvatures make the pressure at point $A$ larger than the pressures at point $B_{1}$ and $B_{2}$, which induces two vapour flows $\left(A B_{1}\right.$ and $\left.A B_{2}\right)$. The $A B_{1}$ flow is weaker because the sideway flow (into and out of page in figure) becomes dominant for its small flow resistance according to the particle tracers' behaviour in their experiments, so a net asymmetric flow in the $A B_{2}$ direction is created ${ }^{[7]}$

Linke et al. ${ }^{[7]}$ also considered other possible mechanisms that influenced the motion of the liquid droplet in their experiments. Firstly, the intermittent contact of the liquid with its substrate at low temperatures will cause vapour to 'burst' from the droplet, leading to an increased acceleration and velocity as already explained in Section 2 . The corresponding pinning effect (contact angle hysteresis) because of the surface wettability has also been analysed by Ok et al. ${ }^{[21]}$. A much higher maximum velocity was achieved by coating the ratcheted substrates with hydrophobic materials where the pinning force was reduced to a large extent. Linke et al. ${ }^{[7]}$ also pointed out that the liquid surface closest to the ratchet peak will obtain a higher temperature, leading to an additional thermocapillary flow.

\subsection{Jet-thrust Momentum Conversion}

Lagubeau et al. ${ }^{[49]}$ disagreed with the viscous mechanism. This is because they observed self-propulsion of solid platelets of dry ice on a ratcheted surface that do not deform, and therefore, do not generate a Laplace pressure differential. They instead proposed that the vapour is rapidly expelled from the Leidenfrost body during evaporation and then rectified by the presence of the ratchets, resulting in a directed vapor flow in the opposite direction to the motion of the dry ice. The speculation was supported by tracer observation and Reynolds number analysis. The magnitude of the Reynolds number of the vapour was estimated by using typical parameters generated from a flat surface (equation 4). Here, $\rho$ is the vapour density $\left(\sim 1 \mathrm{~kg} \mathrm{~m}^{-3}\right), U$ is the radial escape velocity $\left(\sim 1 \mathrm{~m} \mathrm{~s}^{-1}\right), h_{0}$ is the vapour film thickness $(\sim 100 \mu \mathrm{m})$, and $\mu$ is the vapour viscosity at the mean temperature between the substrate and the droplet $\left(10^{-5} \mathrm{~Pa} \mathrm{~s}\right)$. This data gives $R e=10$, confirming the importance of the inertial forces of the flow 
(Lagubeau et al. ${ }^{[49]}$ consider $R e=1$ as the transition between inertial and viscosity dominated vapour flow).

$$
R e=\frac{\rho U h_{0}}{\mu}
$$

Instead, a 'rocket effect' was proposed that explained the forward propulsion of the Leidenfrost body (i.e. Newton's $3^{\text {rd }}$ law of motion). For a quantitative understanding of the propulsion process, they modelled the flow under the assumption of lubrication approximation considering the film thickness was much smaller than the radius of the levitated body, i.e. $h_{0} \ll R$ :

$$
U \sim \frac{h_{0}^{2}}{\mu} \frac{\Delta P}{R}
$$

Here, $U$ is the vapour flow velocity, $h_{0}$ is the vapour film thickness, $\mu$ is the vapour viscosity, $R$ is the radius of levitated body, and $\Delta P$ is the pressure induced by the weight of the Leidenfrost body (equation $6)$, that causes the rectified vapour flow.

$$
\Delta P \sim \rho_{0} H_{0} g
$$

where $\rho_{0}$ and $H_{0}$ are the density and height of the Leidenfrost body respectively. To calculate the vapour flow thickness, $h_{0}$, the evaporated mass was considered:

$$
\dot{M} \sim \rho h_{0} R U
$$

The evaporated mass multiplied by the latent heat corresponds to the energy transferred to the droplet. This can be equated with Fourier's heat conduction law across the vapour film beneath the droplet via a simple energy balance (given here as a scaled representation):

$$
\dot{M} L \sim \frac{\kappa \Delta T R^{2}}{h_{0}}
$$

Combining equations 5-8 then provides a scaled representation of the film thickness, $h_{0}$ (equation 9):

$$
h_{0} \sim\left(\frac{\mu \kappa \Delta T}{L \rho \rho_{0} g H_{0}}\right)^{1 / 4} R^{1 / 2}
$$

In these equations, $\kappa$ is the thermal conductivity of the vapour and $L$ is the latent heat of evaporation. It is assumed that the temperature difference, $\Delta T$, between the bottom of the Leidenfrost body and the ratchet surface is constant. In particular, a flattened large water droplet has a stable height of $\sim 5 \mathrm{~mm}$ ${ }^{[16]}$, making the first term in equation 9 constant at a specific temperature. A coefficient, $C$, was then defined (equation 10) to simplify the relationship between the film thickness and the droplet radius to equation 11.

$$
\begin{gathered}
C=\left(\frac{\mu \kappa \Delta T}{L \rho \rho_{0} g H_{0}}\right)^{1 / 2} \\
h_{0} \sim C^{1 / 2} R^{1 / 2}
\end{gathered}
$$

The driving force for propulsion, $F_{f}$, was finally expressed as $F_{f}=\dot{M} U$. By combining equations 5-11, Lagubeau et al. ${ }^{[49]}$ found that the driving force had a sensitive dependence on the radius of the Leidenfrost body according to equation 12 , where $F_{0}=C^{4} \rho \rho_{0}^{2} g^{2} H_{0}{ }^{2} / \mu^{2}$.

$$
F_{f} \sim F_{0}(R / C)^{3 / 2}
$$

This model suggested an exponent of 1.5 for the power law relationship between the driving force, $F_{f}$, and the droplet radius $R$. To experimentally validate this, Lagubeau et al. ${ }^{[49]}$ used the set up shown in figure $9 \mathrm{a}$. Here, a glass fibre was used to hold a propelling droplet in place, where the elastic tension of the fibre balanced the driving force. The elastic force in response to the horizontal distance, $K \delta$, was 
taken as an equivalent value for the driving force acting on the droplet via the interaction of the vapour and the ratchets. The vertical elastic force was ignored in the analysis. A line of best fit through the experimental data gave a slope of 1.47, seemingly validating the jet thrust mechanism (solid line in figure $9 b)$.

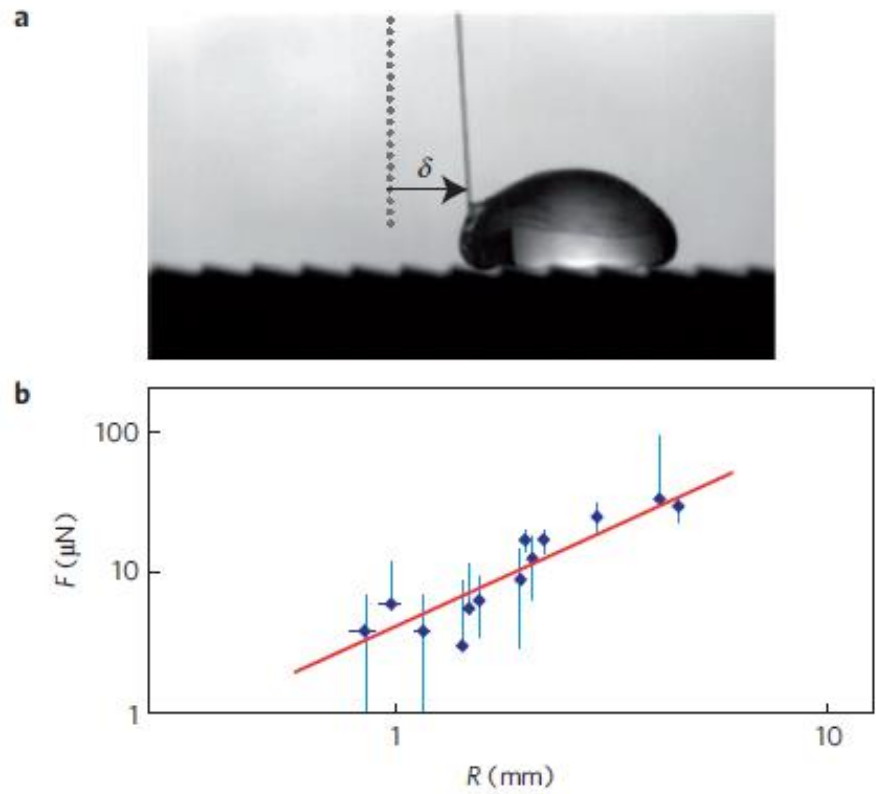

Fig. 9. (a) Force measurement via a glass fire, deflecting the fibre by a distance of $\delta$, (b) Driving force with varying droplet diameter $(R)$ compared to theoretical prediction ${ }^{[49]}$

\subsection{Viscous Stress Induced by Rectified Vapour Flow}

Following the proposed jet thrust mechanism, the same group made a later clarification following the further careful consideration of the vapour flow behaviour beneath the Leidenfrost body. This was deemed to be critical for properly discriminating between the viscous stress drag and the vapour rocket mechanisms. To avoid the experimental error induced by liquid deformation, dry ice was selected as the subject of their investigation ${ }^{[50]}$.

Small glass beads were chosen as tracers to visualise the vapour flow. Tracking the displaced beads confirmed that the vapour flow was isotropic on a flat plate but anisotropic on a ratcheted surface. The majority of the beads had a positive $x$ velocity (self-propelled direction on the order of $10 \mathrm{~cm} \mathrm{~s}^{-1}$ ), though some fell off the step backwards (due to the $\mathrm{AB}_{1}$ vapour flow), a similar conclusion to that of Linke et al. ${ }^{[7]}$. Furthermore, they found that the beads escaped from the ratchets symmetrically perpendicularly to the direct of motion (figure 10), with almost the same velocity as the forward velocity. This effect occurred within each individual ratchet, meaning that a net gas flow in the positive $x$ direction was produced. Consequently, the dry ice platelet above the ratchets was dragged along the descending slope of the teeth. Based on the experiments, they calculated the Reynolds number to be around 0.1 . Here, they defined the Reynolds number using $R e=\left(\rho U^{2} / \lambda\right) /\left(\eta U / h_{0}^{2}\right)$, which compared inertia in the denominator with viscous resistance in the numerator. This result aligned with the viscous stress mechanism proposed by Linke et al. ${ }^{[7]}$. Meanwhile, they clarified that the Reynolds number had been overestimated in their previous jet thrust study where inertial propelling force was considered. 


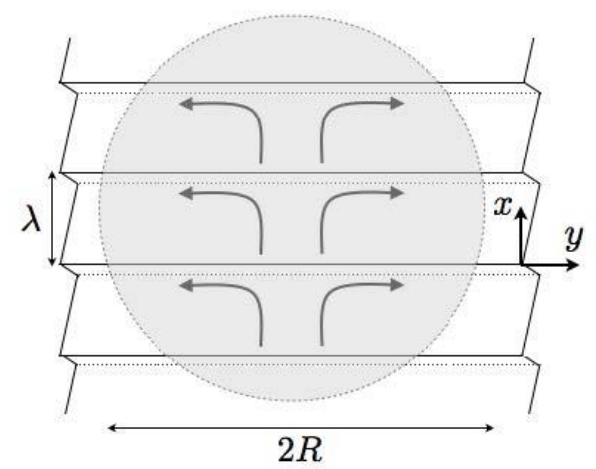

Fig. 10. Vapour flow (arrow direction) rectified by saw-tooth ratchets ${ }^{[50]}$

Following this, the vapour flow velocity was redefined using equation 13 according to the Poiseuille law:

$$
U \sim \frac{h^{2}}{\mu} \frac{d P}{d x}
$$

The pressure was generated from the platelet weight according to equation 14:

$$
P \sim \frac{M g}{A}
$$

Here, $A$ is the 'contact' area of the dry ice platelet that covers the ratchets. With $A_{i}$ representing the corresponding 'contact' area in the $i^{\text {th }}$ ratchet, the shear force per individual ratchet could be expressed as:

$$
f_{f, i} \sim \frac{M g h}{A \lambda} A_{i}
$$

The total driving force was then obtained by adding all the individual driving forces:

$$
F_{f} \sim \sum_{i} f_{f, i}=\frac{M g h}{A \lambda} \sum_{i} A_{i}=\frac{M g h}{\lambda}
$$

The thickness of the vapour flow was much smaller than the ratchet height, $h_{0} \ll \varepsilon$, which resulted in an approximation of $h / \lambda \sim \alpha$, where $\alpha$ is the inclined angle of the teeth relative to the horizontal. Thus the total driving force was simplified slightly to:

$$
F_{f} \sim M g \alpha
$$

For platelets of dry ice, the mass of the disc could be replaced by $M \sim \rho_{0} H_{0} R^{2}$, yielding equation 18:

$$
F_{f} \sim \rho_{0} R^{2} g \alpha H_{0}
$$

This model follows many of the original assumptions made by Dupeux et al. ${ }^{[50]}$, where a different scaling estimation of the 'contact' area was used. The model implies a fully developed vapour flow within the space of each covered ratchet, which may lead to an overestimated vapour thickness. Given that the weak pressure produced by the body weight (order of $10 \mathrm{~Pa}$ ) is much smaller than the surrounding air pressure on the solid surface ( 1 bar), the evaporated vapour may be partially diffused towards the solid surface, but the main flow should be supported by the large amount of compressed vapour underneath, leading to a narrowed vapour flow to produce the viscous force. Following this, the boundary condition for the analytical solution of the effective vapour flow may be changed when considering the component of the accompanied air flow. After all, the model only provides scaled representations for the driving force, providing only a basic parameter indication on the levitated body to be checked by experiments (e.g. radius $R$ or height $H_{0}$ ). 
Experimentally, they calculated the acceleration $(a)$ by following the trajectory of the Leidenfrost body over time, then calculated the corresponding driving force by multiplying by the mass $\left(F_{f}=M a\right)$. To reduce bias in the measurements they gave the Leidenfrost bodies an initial 'negative velocity' or a momentum against the direction of propulsion, so that they would decelerate to zero, then start accelerating in the 'correct' direction. Dry ice platelets were again used in the experiments, and good agreement between equation 18 and the experimental data was observed (figure 11).

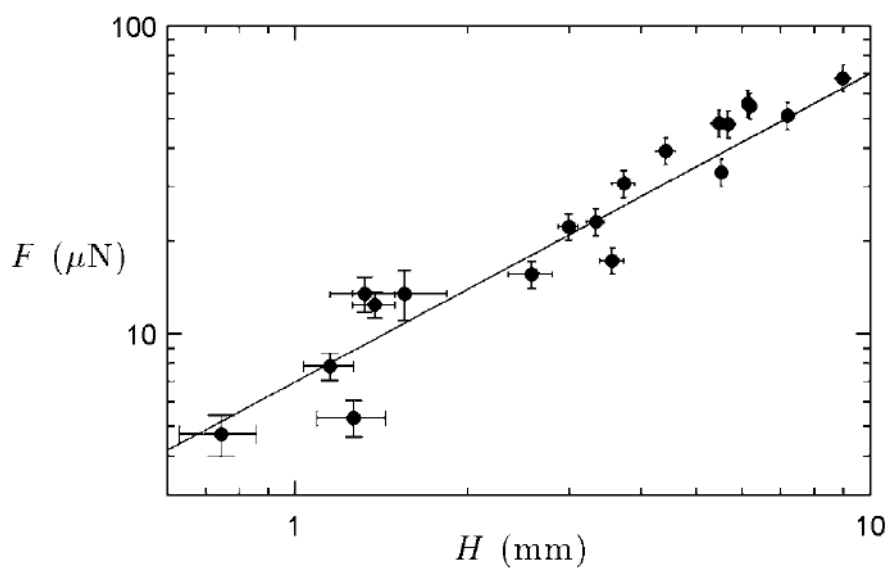

Fig. 11. Force $\left(F_{f}\right)$ acting on dry ice platelet with varying thicknesses $\left(H_{0}\right)$ compared to model prediction $^{[50]}$

\subsection{Additional Considerations for the Pressure-Driven Flow}

Most studies now agree that the evaporated vapour is rectified before dragging the levitated body (whether it be a liquid droplet or solid disc). However, Würger ${ }^{[51]}$ suggested yet an alternative mechanism for the rectified gas flow: thermal creep induced by the strong temperature gradient as indicated by the blue arrows in figure 12. Steffen et al. ${ }^{[52]}$ later 'proved' the insignificance of the thermal flow component via a numerical simulation of the Boltzmann equation, which confirmed the dominant role of the pressure-driven flow. In particular, the pressure-driven flow also explains the observation of 'reversible' Leidenfrost self-propulsion ${ }^{[53,54]}$, where the vapour is directed in the opposite direction because of the different parameter of the ratchets, resulting in motion in the 'opposite' direction as well ('opposite' direction refers to motion from right to left relative to the ratchets in figure 12, whereas the 'correct' direction is from left to right).

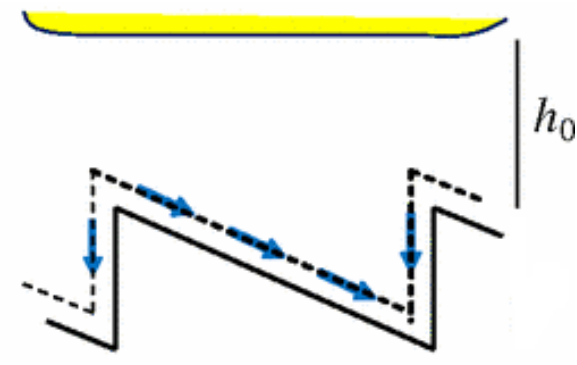

Fig. 12. Directed gas flow (blue arrow) induced by creep flow ${ }^{[51]}$

Based upon the analysis discussed in Section 3.3, more in-depth research has subsequently arisen that aims to give a definitive quantitative description for solid propulsion ${ }^{[55]}$. Here several updates have been made to the model to make it more practical and precise. First, the three-dimensional characteristics have been considered to construct the corresponding Navier-Stokes equations (equation 19). Second, a normal flow velocity, $u_{n}$, is introduced to describe the initial evaporation process as shown in figure 13 (top). The corresponding equation is expressed in equation 20 (deduced from equations 7 and 8). Third, the lateral flow analysis along the groove was added, as shown in figure 12 
(bottom). Finally, the driving force was calculated according to the shear force at the dry ice disc surface, rather than by an average as done previously ${ }^{[50]}$.

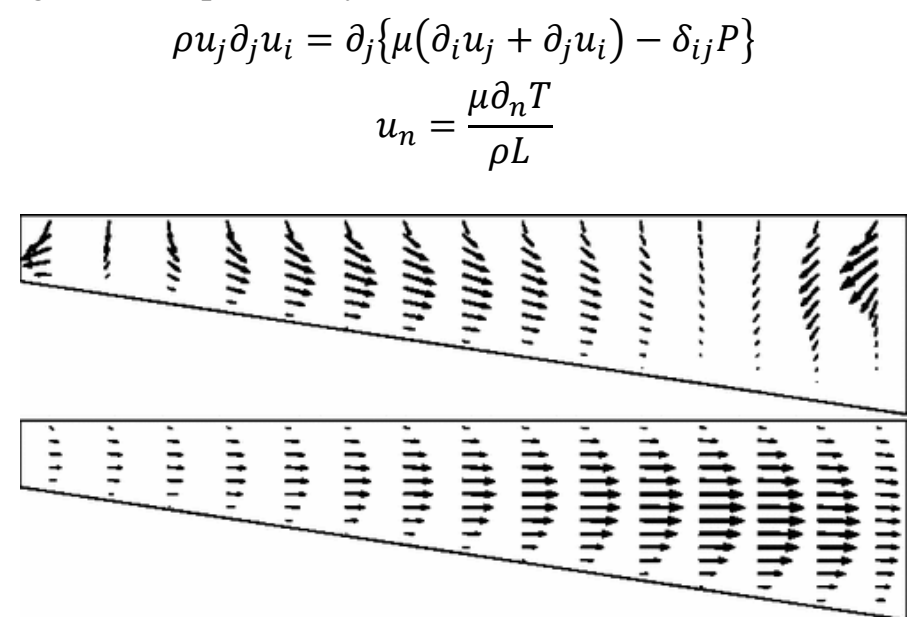

Fig. 13. Rectified vapor flow profiles, with velocities along the slow slope of the ratchet (top) and lateral velocities along the groove (bottom) ${ }^{[55]}$

This study provides a detailed flow field and it has made a breakthrough to supersede the simple scaling analysis previously conducted ${ }^{[50]}$, which may provide a better understanding of the experimental results moving forward; though the error between the experimental data and predictions by the updated model are still under consideration. Note that equations 19 and 20 are only suitable for the Leidenfrost solids at this stage, because the deformation of the liquid will make the analysis more complicated.

\subsection{Friction}

During motion the primary driving force for propulsion is eventually balanced by friction, a consequence of energy loss due to deformation around the ratchets (liquid) and air resistance (liquid and solid), which ultimately gives rise to the terminal velocity of the Leidenfrost bodies on the heated ratcheted surfaces (since the resisting force generated by friction grows as the velocity increases). Some have applied Stokes' law to analyse the frictional drag component exerted by the surrounding air:

$$
M \dot{v}=F_{f}-\beta v
$$

Here, $v$ is the velocity of the droplet and $\beta$ represents the friction coefficient. Integrating equation 21 yields:

$$
v(t)=\frac{F_{f}}{\beta}\left(1-e^{-\beta t / M}\right)
$$

By comparing equation 22 with experimental data of the velocity, the friction coefficient can be obtained, which in turn, enables the driving force to be estimated using equation 21 . By measuring the acceleration $(\dot{v})$.

However, Guillaume et al. ${ }^{[19]}$ estimates that the air resistance is negligible. The friction resulting from the vapour film and the ambient air are scaled by $\left(\mu v / h_{0}\right) R^{2}$ and $\rho v^{2} R^{2}$ respectively. These both have an order of $0.1 \mu \mathrm{N}$, which are 100 times smaller than the driving force. Instead, the main energy loss occurs due to deformation of the liquid around the ratchets and the dissipated kinetic energy as the Leidenfrost liquid droplets strike the ratchets ${ }^{[40]}$, as described in equation 23 . Here, $b_{1} \& b_{2}$ are numerical coefficients to be fitted from experiments. This agrees with the observation that dry ice discs have a much longer acceleration period.

$$
F_{b}=b_{1} \rho_{L} g R^{2} \frac{\varepsilon^{2}}{\lambda}+b_{2} \rho_{L} R^{2} v^{2} \frac{\varepsilon}{\lambda}
$$


This area also warrants further research to better understand the role of friction. This includes the confirmation of the importance of liquid deformation (especially on the smaller scaled ratchets) and further study into how the motion of the droplet (i.e. sliding and/or rotation) influences the friction. For example, recent evidence for rotation on a smooth hot surface is provided by Bouillant et al. ${ }^{[45]}$, where small water droplets were found to self-rotate. Viscous dissipation within these rotating droplets may also need to be taken into account ${ }^{[56,57]}$.

\section{Potential Applications}

Leidenfrost self-propulsion provides several potential benefits for energy harvesting applications. Primarily, these devices function as heat engines, meaning they convert thermal gradients into kinetic energy. The motion on the heated ratcheted surfaces has a predictable direction with a very appreciable terminal velocity compared to other passive liquid transport phenomena. Further, these droplets do not contaminate the substrate as they are suspended by a vapour film, the small value of which also makes the motion near-frictionless $\left(F_{b} \sim 10 \mu \mathrm{N}\right.$ for $\left.R \sim 3 \mathrm{~mm}^{[49]}\right)$.

The simple Leidenfrost cart studied by Ali et al. ${ }^{[20]}$ shows that the transported object does not necessarily needed to be vaporisable. Theoretically, the 'wheels' of this cart can be continuously replenished to enable low-friction transport across heated surfaces. Alternatively, an artificial air flow can also suspend objects and control the propulsion direction; Dan et al. ${ }^{[58]}$ designed a substrate with pores for air injection, eliminating the need to supply thermal energy entirely (the underlying principle of the air hockey table). More recently, a 'side-shooter' design was proposed by Sugioka et al. ${ }^{[59]}$ that could move capillary droplets at higher velocities $\left(\sim 0.15 \mathrm{~m} \mathrm{~s}^{-1}\right)$ using a hydrophobic coating to reduce the friction ${ }^{[60]}$. The side protrusion of the shooter was attached with thin heated films, which deformed the attached liquid droplet resulting in an unbalanced state (as shown in figure 14). The droplet was resultingly propelled away from the heated films with a velocity comparable to that on a ratcheted surface, whilst avoiding the more complex ratcheted surface pattern. The new phenomenon is expected to have potential applications in microfluidics.

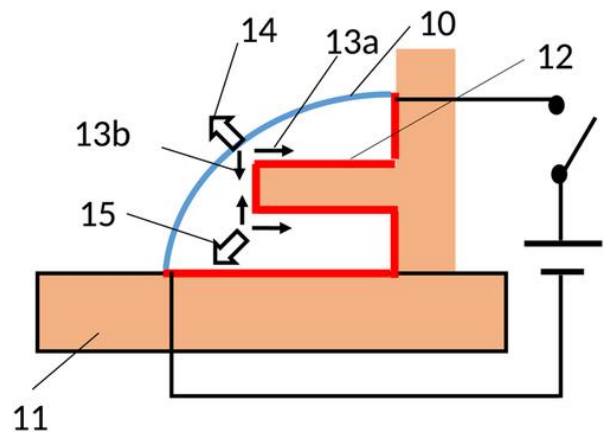

Fig. 14. A Leidenfrost side-shooter $\mid 10$ Water droplet $\mid 11$ Hydrophobic substrate $\mid 12$ Thin-film heater $\mid 13 a \& b$ Surface tension at the corner $\mid 14 \& 15$ Net forces acting on the droplet ${ }^{[59]}$

In another example, a simple mixer was created by paring opposing sets of ratcheted surfaces to induce rotation of a water droplet (as shown in figure 15) ${ }^{[61]}$. Using this device, pigment was observed to spread into the whole liquid droplet rapidly within $0.53 \mathrm{~s}$, whereas no spreading existed in a stationary water droplet. The high rotating speed of the droplet $\left(\sim 0.1 \mathrm{~m} \mathrm{~s}^{-1}\right)$ increases the Reynolds number by a factor of 100 compared with an eletrokinetic phenomenon $\left(\sim 0.001 \mathrm{~m} \mathrm{~s}^{-1}\right)$, suggesting a more competitive way of achieving good mixing. 


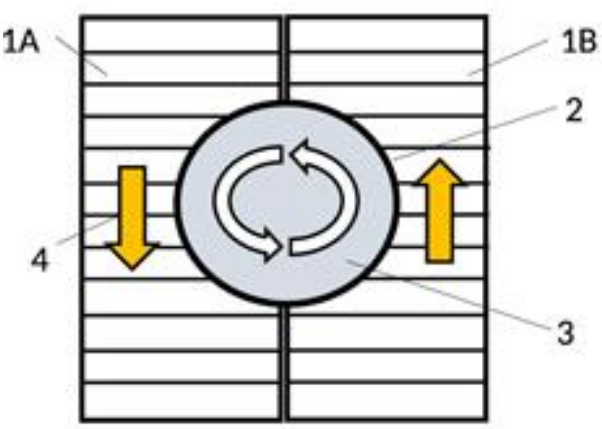

Fig. 15. A Leidenfrost mixer $\mid 1 A \& B$ Ratcheted substrate placed in an opposite direction 2 A ring used to constrain the droplet $\mid 3$ Rotating water droplet $\mid 4$ Ratchets direction ${ }^{[61]}$

The high velocities of the Leidenfrost self-propulsion also makes it promising for the generation of useful energy from waste heat. Gary et al. ${ }^{[62,63]}$ have subsequently attempted to construct a Leidenfrost heat engine for the generation of electricity, though the energy conversion efficiency from phase transition to kinetic motion is still very low $\left(\sim 10^{-6}\right)$ because of the dominant energy consumption required to sustain levitation. It can also be expected that the conversion from kinetic energy to electrical energy will also be very low. Their proposed thermodynamic cycle is shown in figure 16, with a liquid stream rotating on a ratcheted ring as the rotor of the engine. The consumed liquid can be fed by an external pump, which makes the system continuous. The closed thermodynamic cycle is believed to have potential applications in deep space and planetary exploration as the environment there would potentially benefit the energy efficiency of the system (e.g. the vacuum ambient pressure). Further, the typical temperature differences would support different feedstocks (e.g. $\mathrm{H}_{2} \mathrm{O}, \mathrm{CO}_{2}$ or $\left.\mathrm{CH}_{4}\right)$ to support energy generation ${ }^{[63]}$.

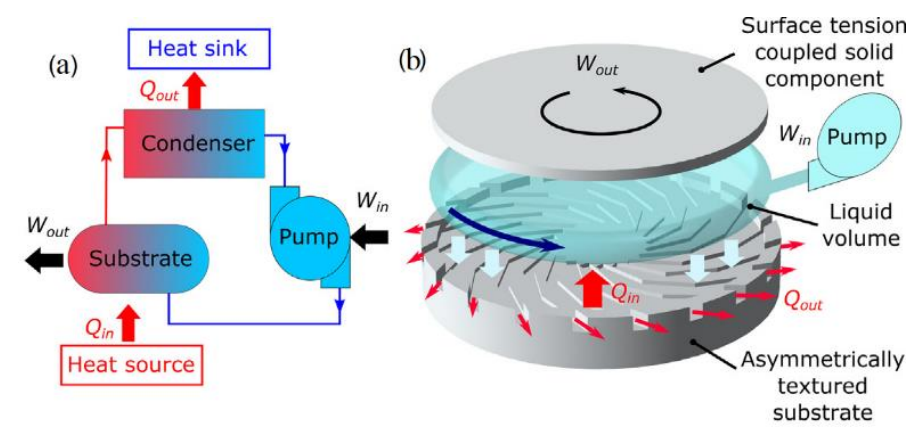

Fig. 16. (a) Energy generation concept. | (b) Experimental realization of a continuously operation Leidenfrost rotor ${ }^{[63]}$

\section{Summary and Outlook}

Liquid droplets (or solid discs) when subjected to the Leidenfrost effect on specially textured ratcheted surfaces exhibit self-propulsion behaviour, provided the droplet/solid covers at least one ratchet period. In general, a liquid droplet may obtain a constant terminal velocity while a solid will keep accelerating because it does not deform, meaning it maintains reduced 'contact' with the surface. Contact hysteresis is a negative factor for the propulsion and can be reduced by coating the surface with hydrophobic additives. At millimetre scales, the terminal velocity of liquid droplets is typically $\sim 0.1 \mathrm{~m} \mathrm{~s}^{-1}$. Faster acceleration occurs at intermediate temperatures, where a transition boiling regime enhances the heat transfer rate. Nucleate boiling at lower temperatures tends to inhibit the droplet propulsion, and higher temperatures lead to the full development of an insulating vapour layer that decreases the evaporation rate (film boiling). By further scaling down the saw-tooth ratchets to micron or sub-micron scales, smaller droplets are able to be propelled on the surface. Meanwhile, the transition boiling regime will be extended and a maximum velocity higher than $0.4 \mathrm{~m} \mathrm{~s}^{-1}$ will be obtained. Further, superhydrophobic coated substrates make it possible to reduce the critical temperature for propulsion even below the boiling point (e.g. $\sim 77{ }^{\circ} \mathrm{C}$ for water), although the velocity tends to decrease in these ultra-low temperature regimes as well. 
In conclusion, more systematic studies need to be conducted to optimise the working conditions to realise higher energy harvesting efficiencies. More detailed theoretical analysis is also necessary because the current viscous mechanism is limited to a scaled model representation, which is not useful for quantification, prediction, or design. Liquid deformation also presents an added complication for the modelling, and further confirmation of droplet rotating and/or sliding behaviours is needed.

One of the potential exciting application envisaged in the literature is electricity generation for space exploration. However, it is obvious that the efficiency of this process will be very low because of the dominant energy consumption required to sustain levitation in comparison to other thermodynamic heat engines. Although there is some scope for improving the operating temperature via a combination of changing the operating temperature, using hydrophobic coatings, and optimisation of the geometries. We envisage that new application will emerge in the future that are better suited to exploit the unusual behaviour of these Leidenfrost self-propulsion devices. 


\section{Nomenclature}

A

$A_{i}$

$a$

$b_{1}, b_{2}$

C

D

$F_{0}$

$F_{b}$

$F_{f}$

$f_{f, i}$

$g$,

$H_{0}$

$h_{0}$

$h$

$i$

K

$L$

$l_{c}$

M

$\Delta p$

$\Delta P$

$R$

$R e$

$r$

$\Delta T$

$T_{T h}$

$t$

$U$

$u_{n}$

V

v

We

$x$

$x(t)$

Contact area of a dry ice platelet covering ratchets $\left(\mathrm{m}^{2}\right)$

Contact area of a dry ice platelet covering one ratchet period $\left(\mathrm{m}^{2}\right)$

Acceleration determined from experimental data $\left(\mathrm{m} \mathrm{s}^{-2}\right)$

Constants determined from experimental data

Constant in equation $11(\mathrm{~m})$

Droplet diameter $(\mathrm{m})$

Constant in equation $12(\mathrm{~N})$

Frictional force that balances the driving force $(\mathrm{N})$

Driving force $(\mathrm{N})$

Driving force in an individual ratchet $(\mathrm{N})$

Gravitational acceleration $\left(\mathrm{m} / \mathrm{s}^{2}\right)$

Thickness of the levitated body (m)

Vapour layer thickness (m)

Levitated height of the body from the surface (m)

Number of ratchets covered by the Leidenfrost body

Elastic constant of the glass fibre $\left(\mathrm{N} \mathrm{m}^{-1}\right)$

Latent heat of evaporation/sublimation of the levitated body $\left(\mathrm{J} \mathrm{kg}^{-1}\right)$

Capillary length of droplet $(\mathrm{m})$

Mass of the levitated body $(\mathrm{kg})$

Pressure difference between the droplet's interior and exterior $(\mathrm{Pa})$

Pressure difference that directs the vapour flow $(\mathrm{Pa})$

Radius of the levitated body (m)

Reynolds number

Local radius of curvature of the droplet $(\mathrm{m})$

Temperature difference between the levitated body and the solid surface $\left({ }^{\circ} \mathrm{C}\right)$

Greek Symbols

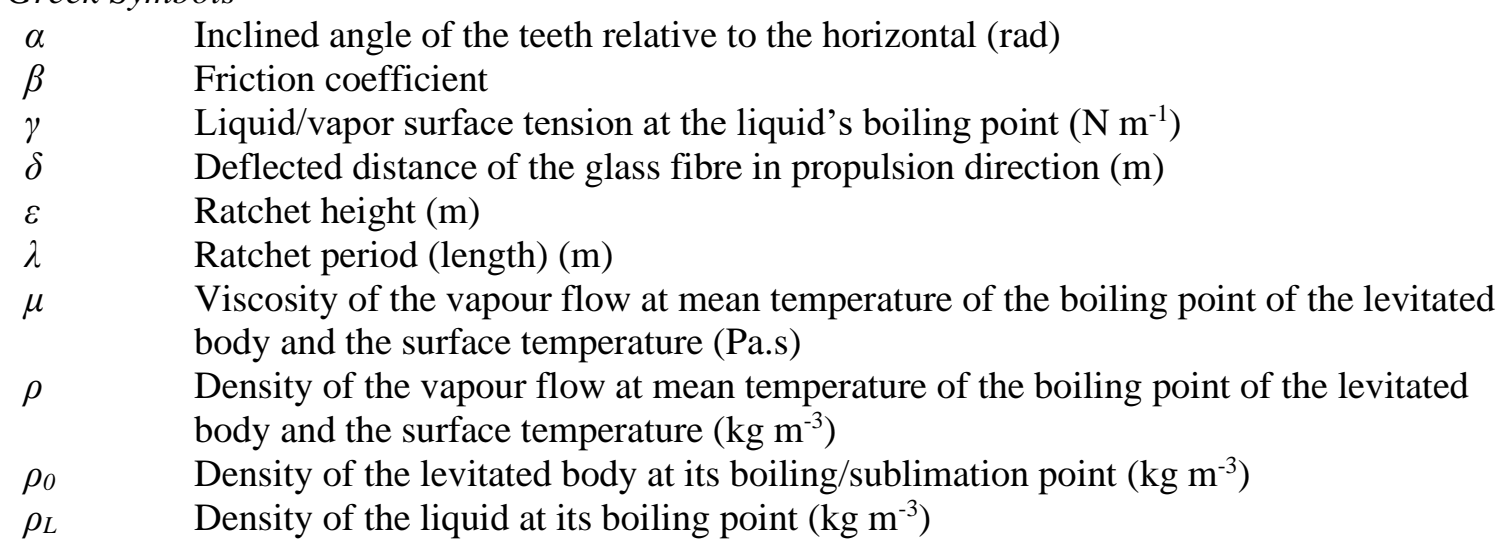




\section{References}

1. Ichimura, K., S.-K. Oh, and M. Nakagawa, Light-Driven Motion of Liquids on a Photoresponsive Surface. Science, 2000. 288(5471): p. 1624-1626.

2. Scriven, L.E. and C.V. Sternling, The Marangoni Effects. Nature, 1960. 187(4733): p. 186-188.

3. Brochard, F., Motions of Droplets on Solid Surfaces Induced by Chemical or Thermal Gradients. Langmuir, 1989. 5(2): p. 432-438.

4. Chaudhury, M.K. and G.M. Whitesides, How to Make Water Run Uphill. Science (New York, N.Y.), 1992. 256(5063): p. 1539-1541.

5. Bico, J. and D. Quéré, Self-Propelling Slugs. Journal of Fluid Mechanics, 2002. 467: p. 101-127.

6. John, K., P. Hänggi, and U. Thiele, Ratchet-Driven Fluid Transport in Bounded Two-Layer Films of Immiscible Liquids. Soft Matter, 2008. 4(6): p. 1183-1195.

7. Linke, H., et al., Self-propelled Leidenfrost Droplets. Physical Review Letters, 2006. 96(15): p. 154502.

8. Reyssat, M., F. Pardo, and D. Quéré, Drops onto Gradients of Texture. EPL (Europhysics Letters), 2009. 87(3): p. 36003.

9. Malvadkar, N., A., et al., An Engineered Anisotropic Nanofilm with Unidirectional Wetting Properties. Nature Materials, 2010. 9(12): p. 1023-1028.

10. Malouin, B.A., et al., Directed Rebounding of Droplets by Microscale Surface Roughness Gradients. Applied Physics Letters, 2010. 96(23).

11. Wu, J., et al., Do Droplets Always Move Following the Wettability Gradient? Applied Physics Letters, 2011. 98(20): p. 204104.

12. Schutzius, T.M., et al., Spontaneous Droplet Trampolining on Rigid Superhydrophobic Surfaces. Nature, 2015. 527(7576): p. 82-85.

13. Buguin, A., L. Talini, and P. Silberzan, Ratchet-Like Topological Structures for the Control of Microdrops. Applied Physics A, 2002. 75(2): p. 207-212.

14. Prakash, M., D. Quéré, and J.W.M. Bush, Surface Tension Transport of Prey by Feeding Shorebirds the Capillary Ratchet. Science (New York, N.Y.), 2008. 320(5878): p. 931-934.

15. Quéré, D., Leidenfrost Dynamics. Annual Review of Fluid Mechanics, 2013. 45(1): p. 197-215.

16. Anne-Laure, B., C. Clanet, and D. Quéré, Leidenfrost Drops. Physics of Fluids, 2003. 15(6): p. 1632-1637.

17. Gottfried, B.S., C.J. Lee, and K.J. Bell, The Leidenfrost Phenomenon: Film Boiling of Liquid Droplets on a Flat Plate. International Journal of Heat and Mass Transfer, 1966. 9: p. 11671187.

18. Wciślik, S., Thermal infrared mapping of the Leidenfrost drop evaporation. 2016.

19. Lagubeau, G., et al., Leidenfrost on a Ratchet. Nature Physics, 2011. 7(5): p. 395-398.

20. Hashmi, A., et al., Leidenfrost Levitation: Beyond Droplets. Scientific Reports, 2012. 2.

21. Ok, J., et al., Propulsion of Droplets on Micro- and Sub-Micron Ratchet Surfaces in the Leidenfrost Temperature Regime. Microfluidics and Nanofluidics, 2011. 10(5): p. 1045-1054.

22. Bradfield, W.S., Liquid-Solid Contact in Stable Film Boiling. Industrial and Engineering Chemistry Fundamentals, 1966. 5(2): p. 200-204.

23. Grounds, A., R. Still, and K. Takashina, Enhanced Droplet Control by Transition Boiling. Scientific Reports, 2012. 2(1).

24. Marín, Á.G., et al., Capillary Droplets on Leidenfrost Micro-Ratchets. Physics of Fluids, 2012. 24(12): p. 122001.

25. Kwon, H.-M., J.C. Bird, and K.K. Varanasi, Increasing Leidenfrost Point Using Micro-Nano Hierarchical Surface structures. Applied Physics Letters, 2013. 103(20): p. 201601.

26. Kim, H., et al., On the Effect of Surface Roughness Height, Wettability, and Nanoporosity on Leidenfrost Phenomena. Applied Physics Letters, 2011. 98(8): p. 083121.

27. Vakarelski, I., U., et al., Stabilization of Leidenfrost Vapour Layer by Textured Superhydrophobic Surfaces. Nature, 2012. 489(7415): p. 274-277.

28. Agapov, R.L., et al., Asymmetric Wettability of Nanostructures Directs Leidenfrost Droplets. ACS Nano, 2014. 8(1): p. 860-867. 
29. Agapov, R.L., et al., Length scale of Leidenfrost Ratchet Switches Droplet Directionality. Nanoscale, 2014. 6(15): p. 9293-9299.

30. Kim, S., M.-W. Moon, and H.-Y. Kim, Drop Impact on Super-Wettability-Contrast Annular Patterns. Journal of Fluid Mechanics, 2013. 730: p. 328-342.

31. Biance, A.-L., et al., On the Elasticity of an Inertial Liquid Shock. Journal of Fluid Mechanics, 2006. 554: p. 47-66.

32. Richard, D., C. Clanet, and D. Quéré, Contact Time of a Bouncing Drop. Nature, 2002. 417(6891): p. 811.

33. Tran, T., et al., Drop Impact on Superheated Surfaces. Physical review letters, 2012. 108(3): p. 036101.

34. Tran, T., et al., Droplet Impact on Superheated Micro-Structured Surfaces. Soft Matter, 2013. 9(12): p. 3272.

35. Chu, K.-H., R. Xiao, and E. Wang, N., Uni-Directional Liquid Spreading on Asymmetric Nanostructured Surfaces. Nature Materials, 2010. 9(5): p. 413-417.

36. Dupeux, G., et al., Propulsion on a Superhydrophobic Ratchet. Scientific Reports, 2014. 4.

37. Bourrianne, P., C. Lv, and D. Quéré, The Cold Leidenfrost Regime. Science Advances, 2019. 5(6).

38. Cousins, T.R., et al., A Ratchet Trap for Leidenfrost Drops. Journal of Fluid Mechanics, 2012. 696: p. 215-227.

39. Dupeux, G., et al., Self-Propelling Uneven Leidenfrost Solids. Physics of Fluids, 2013. 25(5).

40. Dupeux, G., et al., Trapping Leidenfrost Drops with Crenelations. Physical Review Letters, 2011. 107(11): p. 114503.

41. Snezhko, A., E. Ben Jacob, and I. S Aranson, Pulsating-Gliding Transition in the Dynamics of Levitating Liquid Nitrogen Droplets. New Journal of Physics, 2008. 10(4): p. 043034.

42. Adda-Bedia, M., et al., Inverse Leidenfrost Effect: Levitating Drops on Liquid Nitrogen. Langmuir, 2016. 32(17): p. 4179-4188.

43. Janssens, S.D., S. Koizumi, and E. Fried, Behavior of Self-Propelled Acetone Droplets in a Leidenfrost State on Liquid Substrates. Physics of Fluids, 2017. 29(3): p. 32103.

44. Gauthier, A., et al., Self-Propulsion of Inverse Leidenfrost Drops on a Cryogenic Bath. Proceedings of the National Academy of Sciences - PNAS, 2019. 116(4): p. 1174-1179.

45. Bouillant, A., et al., Leidenfrost Wheels. Nature Physics, 2018. 14(12): p. 1188-1192.

46. Scott, R.W., et al., Coupling the Leidenfrost effect and elastic deformations to power sustained bouncing. Nature Physics, 2017. 13(11).

47. Pham, J.T., et al., Spontaneous Jumping, Bouncing and Trampolining of Hydrogel Drops on a Heated Plate. Nature communications, 2017. 8(1): p. 905.

48. Khattak, H.K., S.R. Waitukaitis, and A.D. Slepkov, Microwave Induced Mechanical Activation of Hydrogel Dimers. Soft matter, 2019. 15(29): p. 5804-5809.

49. Lagubeau, G., et al., Leidenfrost on a ratchet. Nature Physics, 2011. 7(5): p. 395-398.

50. Dupeux, G., et al., Viscous Mechanism for Leidenfrost Propulsion on a Ratchet. EPL (Europhysics Letters), 2011. 96(5): p. 58001.

51. Würger, A., Leidenfrost Gas Ratchets Driven by Thermal Creep. Physical review letters, 2011. 107(16): p. 164502.

52. Hardt, S., S. Tiwari, and T. Baier, Thermally Driven Flows Between a Leidenfrost Solid and a Ratchet Surface. Physical review. E, 2013. 87(6): p. 063015.

53. Kruse, C., et al., Self-Propelled Droplets on Heated Surfaces with Angled Self-Assembled Micro/Nanostructures. Microfluidics and Nanofluidics, 2015. 18(5-6): p. 1417-1424.

54. Jia, Z.-H., M.-Y. Chen, and H.-T. Zhu, Reversible Self-Propelled Leidenfrost Droplets on Ratchet Surfaces. Applied Physics Letters, 2017. 110(9): p. 091603.

55. Baier, T., et al., Propulsion Mechanisms for Leidenfrost Solids on Ratchets. Physical Review E, 2012. 87(2): p. 021001.

56. Mahadevan, L. and Y. Pomeau, Rolling Droplets. Physics of Fluids, 1999. 11(9): p. 2449-2453. 
57. Richard, D. and D. Quéré, Viscous Drops Rolling on a Tilted Non-Wettable Solid. EPL (Europhysics Letters), 1999. 48(3): p. 286-291.

58. Soto, D., et al., Air-Levitated Platelets: from Take off to Motion. Journal of Fluid Mechanics, 2017. 814: p. 535-546.

59. Sugioka, H., S. Segawa, and M. Kubota, High-Speed Side-Shooter Using Leidenfrost Phenomena. Journal of Applied Physics, 2019. 125(13): p. 134502.

60. Lafuma, A. and D. Quéré, Superhydrophobic States. Nature Materials, 2003. 2(7): p. 457-460.

61. Sugioka, H., M. Kubota, and S. Segawa, Leidenfrost Mixer. Japanese Journal of Applied Physics, 2019. 58(4): p. 048001.

62. Wells, G.G., et al., A Sublimation Heat Engine. Nature Communications, 2015. 6(1).

63. Agrawal, P., et al., Leidenfrost Heat Engine: Sustained Rotation of Levitating Rotors on TurbineInspired Substrates. Applied Energy, 2019. 240: p. 399-408. 